\title{
Motion Control Point Optimization of Dental Arch Generator
}

\author{
Zhang Yong-De ${ }^{1}$, Gu Jun-Tao ${ }^{1,2}$, Jiang Jin-Gang ${ }^{1 *}$ and Sun Xue-Lian ${ }^{1}$ \\ ${ }^{1}$ Intelligent Machine Institute, Harbin University of Science and Technology, \\ Heilongjiang Harbin 150080, China \\ ${ }^{2}$ Heilongjiang province Electronic \& Information Products Supervision Inspection \\ Institute, Heilongjiang Harbin 150090, China \\ 1*jiangjingang1982@163.com
}

\begin{abstract}
During the manufacture of complete denture, the most important step is to design and generate the dental arch curve which adapts to the requirement of patients according to the jaw arch morphology of them. It is important to study the optimization method of the number and position of control point for the dental arch generator. This paper focuses on of the multi-manipulator tooth-arrangement robot. Quantitative model of the dental arch generator is established. On the basis of motion analysis of the dental arch generator, objective function, multivariate design and constraint function of control point optimization of dental arch generator is determined. Control point number of the dental arch generator is optimized. Single point error method of the dental arch generator is presented to realize the control point position optimization of the dental arch generator. Simulation results verify the feasibility of control point optimization method.
\end{abstract}

Keywords: dental arch generator; motion analysis; motion control point; single point error method

\section{Introduction}

After human natural dentition is lost completely, craniofacial morphology changes, vocal and masticatory function is seriously affected. For recovering craniofacial morphology and physiological functions of edentulous patients and protecting health of craniofacial tissues mandibular joint, complete denture is needed timely for edentulous patients [1-3]. So during the manufacture of complete denture, the most important step is to design and generate the dental arch curve which adapts to the requirement of patients according to the jaw arch morphology of them [4-6]. In the orthodontic treatment, individual dental arch which is consistent with the dental arch form of patient is also needed [7, 8]. The traditional way of acquiring the dental arch curve form is based on manual operation, which will randomly bring a lot of errors caused by human factors. The dental arch generator is the key component of the system of multi-manipulator tooth-arrangement robot, and the dental arch curve is acquired by its distortion. These not only realize quantification, standard and automation of acquiring dental arch curve, but also improve its efficiency and accuracy. Also whether the curve produced by the generator tallies with the real one is the important reason of the complete denture error [9-11]. So it is important to study the optimization method of the number and position of control point for the dental arch generator.

This paper focuses on of the multi-manipulator tooth-arrangement robot. Quantitative model of the dental arch generator is established. On the basis of motion analysis of the dental arch generator, objective function, multivariate design and constraint function of control point 
optimization of dental arch generator is determined. Control point number of the dental arch generator is optimized. Single point error method of the dental arch generator is presented to realize the control point position optimization of the dental arch generator.

\section{Mathematical Model of the Dental arch Generator}

Prosthodontics is a qualitative science, which mainly depends on experiences and accumulated skilled. Qualitative descriptions in prosthodontics and orthodontics can not exactly record the experiences of a dentist. In order to improve this situation, some experts of prosthodontic have thoroughly studied geometric shapes of mandibular and dental arch. There are mainly two mathematical models to describe these shapes: the beta function model [12] and power function model [13]. In this paper, the power function model is accepted to represent a jaw arch or dental arch, because it is relatively mature and suitable for majority of Chinese people's arch character. Descriptions between edentulous mandibular arch and artificial dentition (dental arch) are similar, and can be described as follows:

$$
y=\alpha x^{\beta} \quad x \geq 0
$$

Where $\alpha$ and $\beta$ are character parameters of jaw arch or dental arch, which are calculated as follows:

$$
\left\{\begin{array}{l}
\beta=\sigma(S / W-\mu L / W)^{\tau} \\
\alpha=L / W^{\beta}
\end{array}\right.
$$

Where $S, W$ and $L$ are arc length, width and height of the half mandibular or dental arch, and $\sigma, \mu$, and $\tau$ are constants.

Model of the dental arch generator is got by offsetting the dental arch $58.5 \mathrm{~mm}$ outward along the normal direction of its function.

Let the curve function is as follows:

$$
\boldsymbol{C}(t)=[x(t), y(t)] \quad t \in[0,1]
$$

As the excursion curve is at the outer of the dental arch curve, and the value of excursion is $d=-58.5 \mathrm{~mm}$, so the parameter function of dental arch generator in the coordinate system of dental arch curve is as follows:

$$
\boldsymbol{C}_{0}(t):\left\{\begin{array}{l}
x_{0}(t)=t+\frac{58.5 \cdot \alpha \cdot \beta \cdot t^{\beta-1}}{\sqrt{1+\alpha^{2} \cdot \beta^{2} \cdot t^{2 \beta-2}}} \\
y_{0}(t)=\alpha \cdot t^{\beta}-\frac{58.5}{\sqrt{1+\alpha^{2} \cdot \beta^{2} \cdot t^{2 \beta-2}}}
\end{array}\right.
$$

Where $t$ is horizontal coordinate of the dental arch curve.

The dental arch parameters $S, W$ and $L$ of the dental arch curve are substituted into the equations (2), then $\alpha$ and $\beta$ are obtained. Put $\alpha$ and $\beta$ into equations (4), the parameter function of dental arch generator in the coordinate system of dental arch curve is obtained. 


\section{Control Point's Optimization Theory of the Dental arch Generator}

\subsection{Motion Analysis of the Dental Arch Generator}

The structure of the dental arch generator of tooth-arrangement robot is as shown in Figure 1. It is made of flexible material. According to optimal theory using multi-objective function, we can conclude that control number and position of the dental arch is best fitted. Control points on the dental arch generator are controlled by slipway mechanisms to create a dental arch curve that matches the one from a patient's oral cavity. And the point in the symmetrical line is fixed, In order to realize each control point to get any position in the plane, each control point needs two motors to drive, the dental arch generator are controlled by slipway mechanisms to change the control point's position, then the form of the dental arch generator is changed to match the one from a patient's oral cavity.

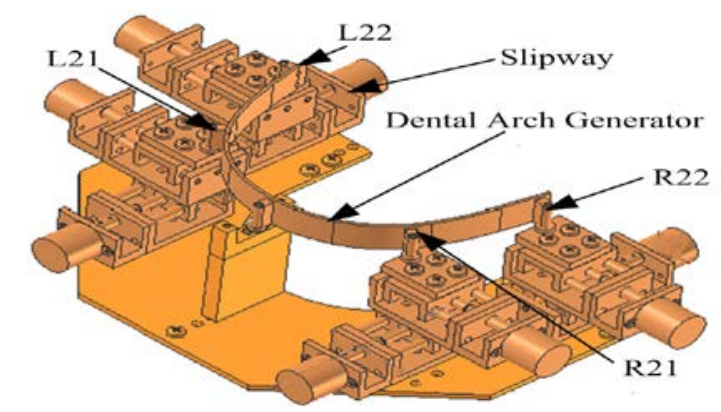

Figure 1. Structure of Dental Arch Generator

\subsection{Objective Function of Control Point Optimization of the Dental Arch Generator}

Control point's optimization problem of the dental arch generator can be expressed as a mathematical function.

$$
\begin{array}{ll}
f(x) \rightarrow \min & x \rightarrow\left\{x_{1}, x_{2}, \cdots, x_{n}\right\} \\
h_{k}(x)=0 & k=1,2, \cdots, p \\
g_{i}(x) \geq 0 & i=1,2, \cdots, m
\end{array}
$$

Where $f(x)$ is the objective function; $X$ is the design variables, it is n-dimensional vector; $h_{k}(x), g_{i}(x)$ is the equality and inequality constraint functions respectively.

The purpose of optimization is to make error value between deformed curve of the dental arch generator and the dental arch minimize. That is:

$$
W(X) \rightarrow \min
$$

\subsection{Variable Design of Control Point Optimization of the Dental Arch Generator}

Generally, the fewer design variables, the more simple optimization, therefore, the choice of the design variables must be carefully [14]. We define the error between deformed curve of the dental arch generator and the dental arch as calculation error when fitting dental arch with dental arch generator. There have many factors to affect the error value, such as the number of fitting points and the coordinates, material, force, size and shape of dental arch generator. We assume that the force on each part of the dental arch generator is equally, the material has 
been selected. Selected the number $n$ of fitting points, coordinate value $x_{i}$ and $y_{i}(1 \leq i \leq n)$ is selected as design variables.

\subsection{Constraint Function of Control Point Optimization of the Dental Arch Generator}

3.4.1. Performance Constraint: Performance constraint can also be called functional constraint, it is a constraint established by the functional requirements, and the design object should meet functional requirements [15]. For structural optimization problems, performance constraint mainly considers the requirements of structural strength; stiffness and vibration mode. In this problem, we needn't to define the performance constraint, only need consider the shape problem.

3.4.2. Boundary Constraint: Boundary constraint can also be called interval constraint, this paper requires arrange 14 dentures, plus a fixed point, it totals 15 points. So, we define $3 \leq n \leq 15$. According to statistics result of dentists, the maximum value of the human dental arch width is $52 \mathrm{~mm}$, so we define the range of horizontal coordinate is $-26 \mathrm{~mm} \leq x_{i} \leq 26 \mathrm{~mm}(1 \leq i \leq n)$.

From the above all, the mathematical model for the optimization problem of the dental arch generator fitting dental arch is:

$$
W\left(n, x_{i}, y_{i}\right) \rightarrow \min \quad i \in[1, n]
$$

\section{The Optimized Design of Control Point Number for the Dental Arch Generator}

It is very important to select the number of control points when fitting dental arch with dental arch generator. This directly affects the error between fitting arch curve and the real dental arch.

Fitting level of two curves can be evaluated by many indexes. Select the area (Figure 2 shaded area) surrounded by two curves as an evaluation index, using the trapz $(x, y)$ function in MATLAB to calculate the area.

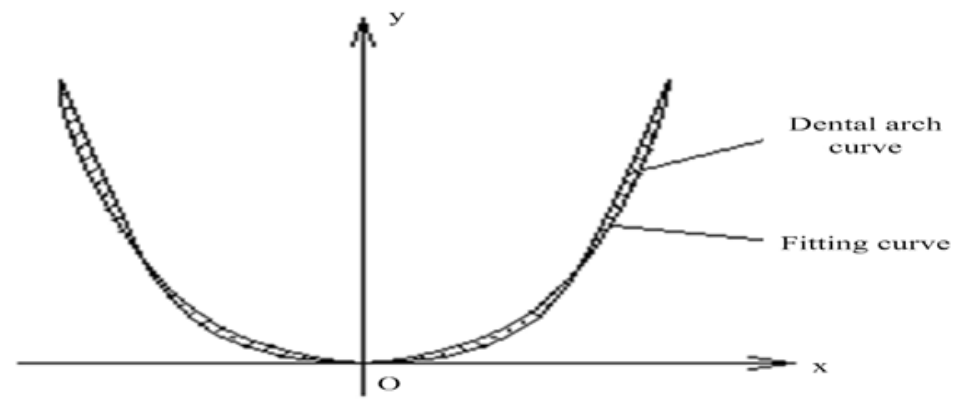

Figure 2. The Comparison of Dental Arch Curve and Fitting Curve

Select a dental arch, the equation is:

$$
y=0.003166|x|^{2.8442}
$$

The selected points include the origin of coordinates, two endpoints of the curve, and some point within the range about $(-26 \mathrm{~mm}, 26 \mathrm{~mm})$. Because of the ranges of design variables is $n$, we need to compare the calculated area error values with selecting different values $n$. 
The curve is fitted with the least squares method, and the coordinates of point and the error value are calculated by MATLAB function program in different number of fitting points. To simplify the complexity of the program, the fitting points uniformly distributed on the horizontal axis.

When the number of fitting points(control points) is changed, two fitting curve is shown in Figure 3. The Figure 3 reflects the fitting relationship between the fitting curve and the dental arch when fitting points number is from 15 to 3 . It can be seen from the Figure 3 that the two curves are quite separated and the degree of agreement is very bad on $n=3$. It proves that the three fitting point cannot meet the design requirements, but the fitting effect is relatively good in other cases.

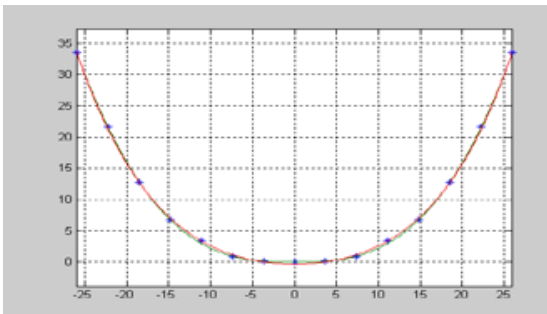

a) $n=15$

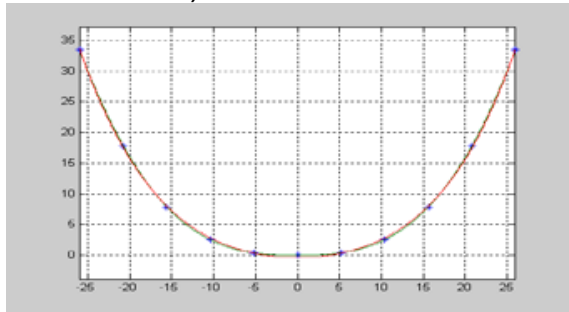

c) $\mathrm{n}=11$

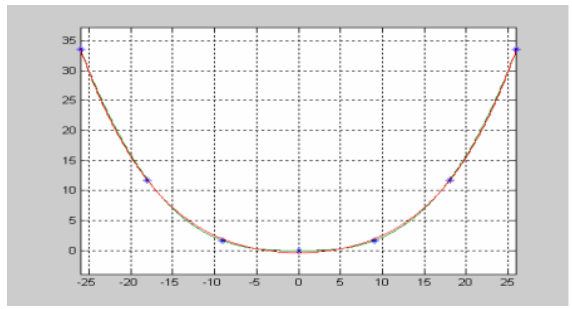

e) $n=7$

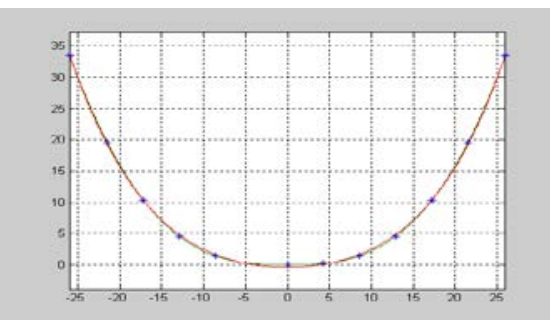

b) $n=13$

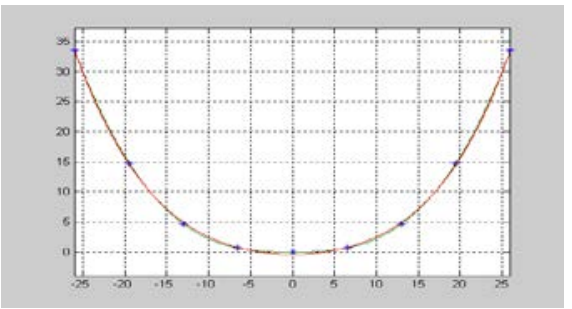

d) $n=9$

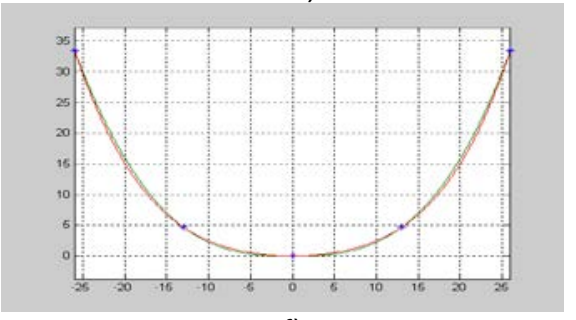

f) $n=5$

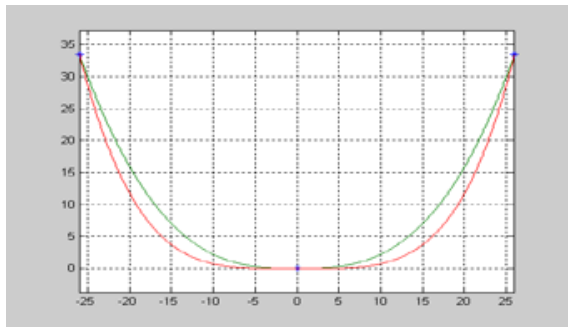

g) $n=3$

Figure 3. Comparison of Fitting Situation with Different Fitting Point Number

It can be known from the results that the error is 104.7312 on $n=3$, the error is significantly reduced to 18.8488 on $n=5$, The error basically stable at 10 to 11 when $n$ is gradually increasing; while the error began to rise again on $n=15$. 
Therefore, the error is minimized on $n=13$. But choices the values of $n$ not only need to make the error as small as possible, but also to make the mechanism has the practical significance. If we choose $n=13,12$ movement mechanism is needed, it will be difficult to achieve at the limited institutional space. We choose $n=5$, only 4 movement mechanism is needed.

Consider the size of error and the feasibility of Program, the number of fitting point are determined to be 5 .

\section{Control Point Position's Optimal Computation of the Dental Arch Generator}

According to optimal result using multi-objective function, we conclude that the dental arch is best fitted by five control points. Because the point in the symmetrical line is fixed, the rest four control points on the dental arch generator are controlled by slipway mechanisms. Two control points locate at the two end of the dental arch generator, and a fixed point locates at the coordinate system's origin. So another two points' position should be analyzed and determined.

\subsection{Definition of Single Point Error of the Dental Arch Generator}

Select the area surrounded by two curves to measure the error when calculating the number of fitting points. This way can get the sum of error of any points on the horizontal axis, but it cannot get the size of the error on each point. We do not want the sum of the error is relatively small, while the error on some of other point is relatively large. Therefore, we need to comprehensive analysis each situation, not only to measure the area surrounded by two curves, but also to measure the error on a single point by calculating.

For analyzing the control's precise, the maximum value of single point along the normal direction, that is the maximum value of any point's error along the normal direction between experimental curve and theoretical curve of dental arch generator, is regarded as the error evaluation criterion. Calculation diagram of single point error of the dental arch generator is shown in Figure 4. Point A denotes any point on theoretical curve of the dental arch generator, and we draw the law line through point $\mathrm{A}$, the law line crosses with the experimental curve at point $\mathrm{B}$. The length of line segment $\mathrm{AB}$ is just the single point error.

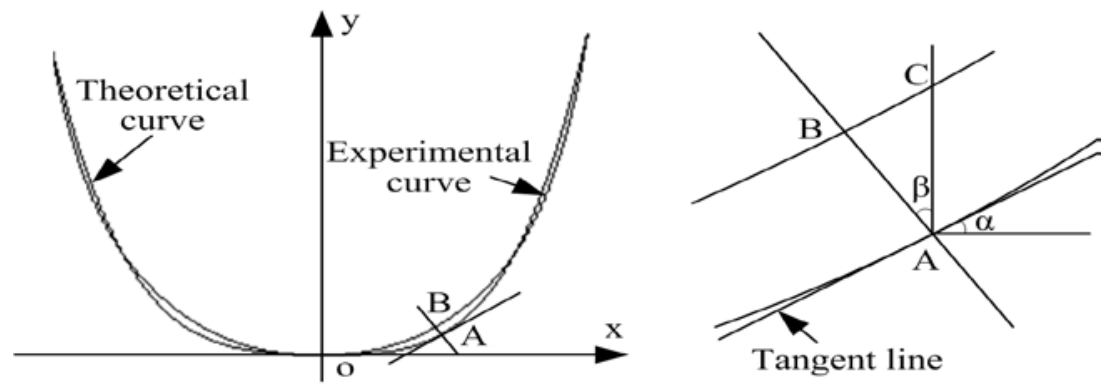

Figure 4. Calculation Diagram of Single Point Error of the Dental Arch Generator

\subsection{Calculation of Single Point Error of the Dental Arch Generator}

The calculation method is as follows: according to the coordinate values of point $\mathrm{A}$, we can obtain the tangent-slope and the law line function at point $\mathrm{A}$, and the coordinate value of 
point $\mathrm{B}$ which is the intersection point of the law line and the experimental curve. Then the length of line segment $A B$ is obtained. However the calculation is complex, in order to simplify the calculation, we can approximate the length of line segment AB. Draw the vertical line of $\mathrm{X}$-axis at point $\mathrm{A}$, and this vertical line intersect with the theoretical curve at point $\mathrm{C}$. According to the theoretical curve function, the coordinate values of point $\mathrm{C}$ can be obtained, and then we can obtain the length of line segment AC. We think the tangent line of theoretical curve at point $\mathrm{A}$ is approximately parallel to the tangent line of experimental curve at point $\mathrm{B}$, so $\angle \alpha=\angle \beta$. Let $K$ denote the tangent-slope, then $K=\tan \alpha$, so the length of line segment $\mathrm{AB}$ is as follows:

$$
|A B|=\frac{1}{\sqrt{1+K^{2}}}|A C|
$$

\subsection{Position Calculation of Control Point based on Single Point Error}

When the abscissa $x=15.0$, the minimum value of square error is 0.1621 , so we selected the abscissa position of fitting point is 15.0. Comparing the fitted curve and the dental arch in this case, the maximum value of error is $0.4026 \mathrm{~mm}$ which is the square root of 0.1621 . Figure 5 shows the fitting curve and an error when $\mathrm{x}=15.0$.
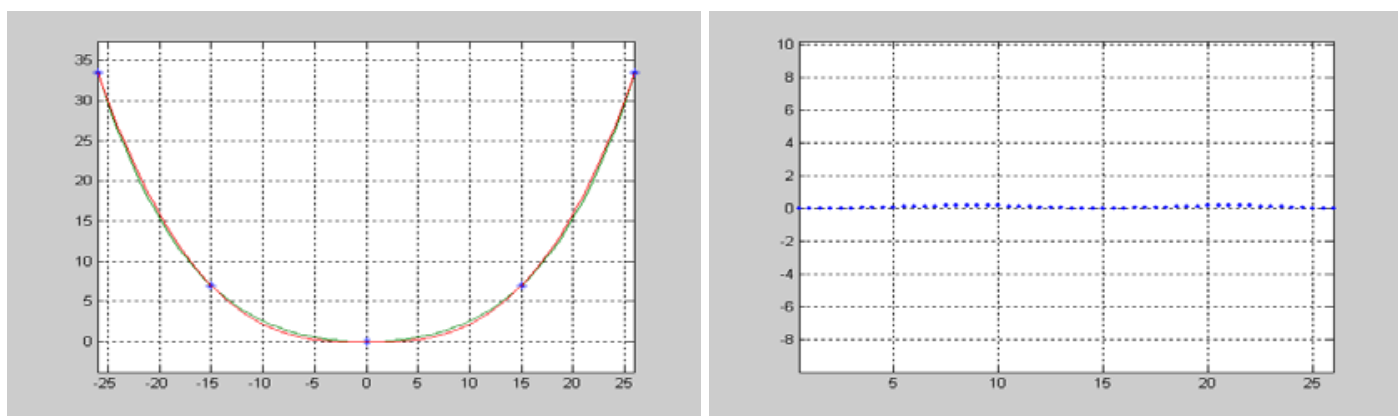

Figure 5. The Curve and Error while the Fitting Point Number is $\mathbf{5}$

The above analysis of the value of error at single-point, the value of error also needs to be measured by the area surrounded by two curves.

When $x=16.5 \mathrm{~mm}$, the minimum value of area is $15.3342 \mathrm{~mm}^{2}$, so we select the number of fitting point is $x=16.5 \mathrm{~mm}$. The value of $x$ calculated in previous part basically consistent with this data. So we select the range of fitting point is $x \in[15,16.5]$.

To realization the organization, we should select the position of a point on the spring board, this position should be to determine, so we should select a right point. $x=15 \mathrm{~mm}$ can be got by approximate calculation, but $x=16.5 \mathrm{~mm}$ is a relatively precise data. So we selected the coordinates of fitting points to $x=16 \mathrm{~mm}$ which is closer to $x=16.5 \mathrm{~mm}$.

\section{Conclusion}

During the manufacture of complete denture, the most important step is to design and generate the dental arch curve which adapts to the requirement of patients according to the jaw arch morphology of them. It is important to study the optimization method of the number and position of control point for the dental arch generator.

(1) Quantitative model of the dental arch generator is established. On the basis of motion analysis of the dental arch generator, objective function, multivariate design and constraint function of control point optimization of dental arch generator is determined. 
(2) Control point number of the dental arch generator is optimized. Consider the size of error and the feasibility of Program, the number of control point are determined to be 5 .

(3) Single point error method of the dental arch generator is presented to realize the control point position optimization of the dental arch generator. Simulation results show that the coordinates of control points is $\mathrm{x}=16 \mathrm{~mm}$ which is tallies with the real dental arch curve.

\section{Acknowledgements}

We are extremely grateful to the anonymous referees for their most insightful and constructive comments, which have enabled us to improve the manuscript significantly. The authors are also grateful to the Editor in Chief and the technical editor for reviewing and editing the paper. This research were supported by the National Natural Science Foundation of China (Grant No. 51205093, 50675054) and the National High Technology Research and Development Program of China (Grant No. 2013AA0408).

\section{References}

[1] N. Michiko, I. Yasutomo, S. J. Seong, H. O. Sang, G. Lian and T. Yasuhiko, “A morphological study on the classification of maxillary dental arches”, Okajimas Folia Anatomica Japonica, vol. 81, no. 1, (2004), pp. 514.

[2] T. Kageyama, R. G. Dominguez, J. Vigoriyo and T. Deguchi, “A morphological study of the relationship between arch dimensions and craniofacial structures in adolescents with Class II Division 1 malocclusions and various facial types”, American Journal of Orthodontics and Dentofacial Orthopedics, vol. 129, no. 3, (2006), pp. 368-375.

[3] K. Alemzadeh, R. A. Hyde and J. Gao, "Prototyping a robotic dental testing simulator", Proceedings of the Institution of Mechanical Engineers, Part H: Journal of Engineering in Medicine, vol. 221, no. 4, (2007), pp. 385-396.

[4] Y. D. Zhang, Z. F. Zhao, P. J. Lü, Y. Wang, R. J. Song and J. L. Lu, "Robotic system approach for complete denture manufacturing”, IEEE/SAME Transactions on Mechatronics, vol. 7, no. 3, (2002), pp. 392-396.

[5] P. J. Lü, Y. Wang and G. Z. Li, "Development of a system for robot aided teeth alignment of complete denture”, Chinese Journal of Stomatology, (in Chinese), vol. 36, no. 2, (2001), pp. 139-142.

[6] Y. D. Zhang, J. G. Jiang, P. J. Lü and Y. Wang, "Study on the multi-manipulator tooth-arrangement robot for complete denture manufacturing”, Industrial Robot: An Internationnal Journal, vol. 38, no. 1, (2011), pp. 2026.

[7] Y. D. Zhang, J. G. Jiang, T. Liang and W. P. Hu, "Kinematics modeling and experimentation of multimanipulator tooth-arrangement robot for full denture manufacturing”, Journal of Medical Systems, vol. 35, no. 6, (2011), pp. 1421-1429.

[8] A. P. Valenzuela, M. A. Pardo and S. Yezioro, "Description of dental arch form using the Fourier series", The International Journal of Adult Orthodontics and Orthognathic Surgery, vol. 17, no. 1, (2002), pp. 59-65.

[9] J. M. Battagel, "Individualized catenary curves: their relationship to arch form and perimeter", British Journal of Orthodontics, vol. 23, (1996), pp. 21-28.

[10] E. A. Begole and R. C. Lyew, "A new method for analyzing change in dental arch form”, American Journal of Orthodontics and Dentofacial Orthopedics, vol. 113, no. 4, (1998), pp. 394-401.

[11] J. Jingang and Z. Yongde, "Motion planning and synchronized control of the dental arch generator of the tooth-arrangement robot”, The International Journal of Medical Robotics and Computer Assisted Surgery, vol. 9, no. 1, (2013), pp. 94-102.

[12] H. Noroozi, "Re: the form of human arch", The Angle Orthodontist, vol. 70, no. 4, (2000), pp. 271-275.

[13] H. Noroozi, T. H. Nik and R. Saeeda, “The dental arch form revisited”, The Angle Orthodontist, vol. 71, no. 5, (2001), pp. 271-275.

[14] T. Trivino, D. F. Siqueira and M. A. Scanavini, "A new concept of mandibular dental arch forms with normal occlusion”, American Journal of Orthodontics and Dentofacial Orthopedics, vol. 133, no. 1, (2008), 10.e15-22.

[15] S. Mutinelli, M. Manfredi and M. Cozzani, "A mathematic-geometric model to calculate variation in mandibular arch form”, The European Journal of Orthodontics, vol. 22, no. 2, (2000), pp. 113-125. 